\title{
Vertical Diplopia as a Major Manifestation of Acute Peripheral Vestibulopathy
}

\author{
Ji-Yun Park ${ }^{a}$ \\ Jee-Hyun Kwon ${ }^{a}$ \\ Wook-Joo Kim ${ }^{\mathrm{a}}$ \\ Sun-Young Kim ${ }^{\mathrm{a}}$ \\ Eun-Mi Lee ${ }^{a}$ \\ Hui-Jun Yang ${ }^{a}$ \\ Byeong-su Park ${ }^{\mathrm{a}}$ \\ Kwang-Dong Choi ${ }^{b}$ \\ aDepartment of Neurology, \\ Ulsan University Hospital, \\ University of Ulsan College of Medicine, \\ Ulsan, Korea \\ ${ }^{b}$ Department of Neurology, \\ Pusan National University Hospital, \\ Pusan National University \\ School of Medicine, Busan, Korea
}

Dear Editor,

Acute peripheral vestibulopathy (APV) is characterized by an episode of acute, prolonged vertigo caused by peripheral vestibular tone imbalance, and patients typically experience severe vertigo (spinning/swaying sensation), imbalance, nausea, and vomiting. ${ }^{1}$ Clinical and laboratory evaluations have shown various combinations of vestibular imbalance, including mixed horizontal-torsional nystagmus beating away from the lesion side and ipsilesional caloric paresis, abnormalities in head impulse test (HIT) results, the ocular tilt reaction, and subjective visual vertical (SVV) tilt, and decreased vestibular-evoked myogenic potentials (VEMPs). ${ }^{2}$ However, the diagnosis is occasionally challenging due to atypical manifestations, particularly in APV patients in which horizontal canal function is spared. ${ }^{3-6}$ We report a patient presenting with acute vertical diplopia as a major manifestation of APV.

A 63-year-old female presented with vertical diplopia and mild imbalance that first appeared 3 days previously. She denied any preceding upper respiratory infection or diarrhea. An examination revealed a skew deviation with vertical displacement in the prism cover test (2 prism diopters of hypertropia in the left eye). There was no spontaneous nystagmus or head tilt. The results of bedside horizontal HITs were normal. She veered to the left while tandem walking. Other neurological examinations produced unremarkable finding. The Hess screen test showed a skew deviation with a hypertropic left eye (Fig. 1A), and fundus photography revealed extorsion $\left(20^{\circ}\right)$ of the right eye and intorsion $\left(2^{\circ}\right)$ of the left eye [pathological ocular torsion is defined when the eye shows any intorsion $\left(<0^{\circ}\right)$ or extorsion of $>12.6^{\circ}$ ] (Fig. 1B). The patient had rightward SVV tilt with binocular viewing $\left(10.0^{\circ}\right.$; normal range $-3.0^{\circ}$ to $3.0^{\circ}$, a negative value indicates a counterclockwise tilt). Video HITs disclosed decreased a vestibulo-ocular reflex (VOR) gain with corrective catch-up saccades of the right posterior semicircular canal (PC) (Fig. 1C), while bithermal caloric tests and air-conducted cervical VEMP (Fig. 1D) tests produced symmetrical results. Brain MRI scans including DWI with 3-mm-thick sections performed at 3 and 10 days after the onset of symptoms were unremarkable. The symptoms rapidly resolved 3 days later, and the findings for video HITs, ocular torsion, and SVV tilt returned to normal.

Skew deviation is vertical misalignment of the eyes caused by a supranuclear disorder involving the otolith-ocular pathways. The phenomenon is mostly seen in the brainstem or cerebellar lesions, and it is one of the important signs suggesting stroke in a part of the head impulse, nystagmus, and test of skew (HINTS). ${ }^{7}$ There are rare cases of skew deviation also being observed in the acute stage following unilateral peripheral vestibular surgery, such as labyrinthectomy or vestibular neurectomy, or vestibular neuritis. ${ }^{4,8}, 9$

Our patient developed acute vertical diplopia as a major manifestation of APV. A neurootological evaluation produced findings suggestive of the selective involvement of the unilateral PC and utricle. Remarkably, there were no typical vestibular symptoms or signs of APV such as vertigo with nausea/vomiting, spontaneous nystagmus, or abnormality in hori-

@ This is an Open Access article distributed under the terms of the Creative Commons Attribution Non-Commercial License (https://creativecommons.org/licenses/by-nc/4.0) which permits unrestricted non-commercial use, distribution, and reproduction in any medium, provided the original work is properly cited. 
zontal HITs. Previous cases with skew deviation following unilateral peripheral vestibulopathy presented with predominantly vestibular symptoms or signs. ${ }^{3,10}$ Patients with acute

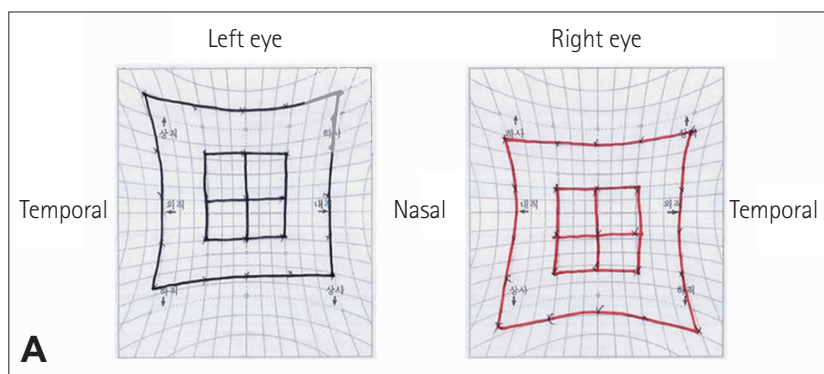

vertical diplopia due to skew deviation with intact horizontal semicircular canal function generally require a workup for brainstem or cerebellar stroke. However, the normal results of
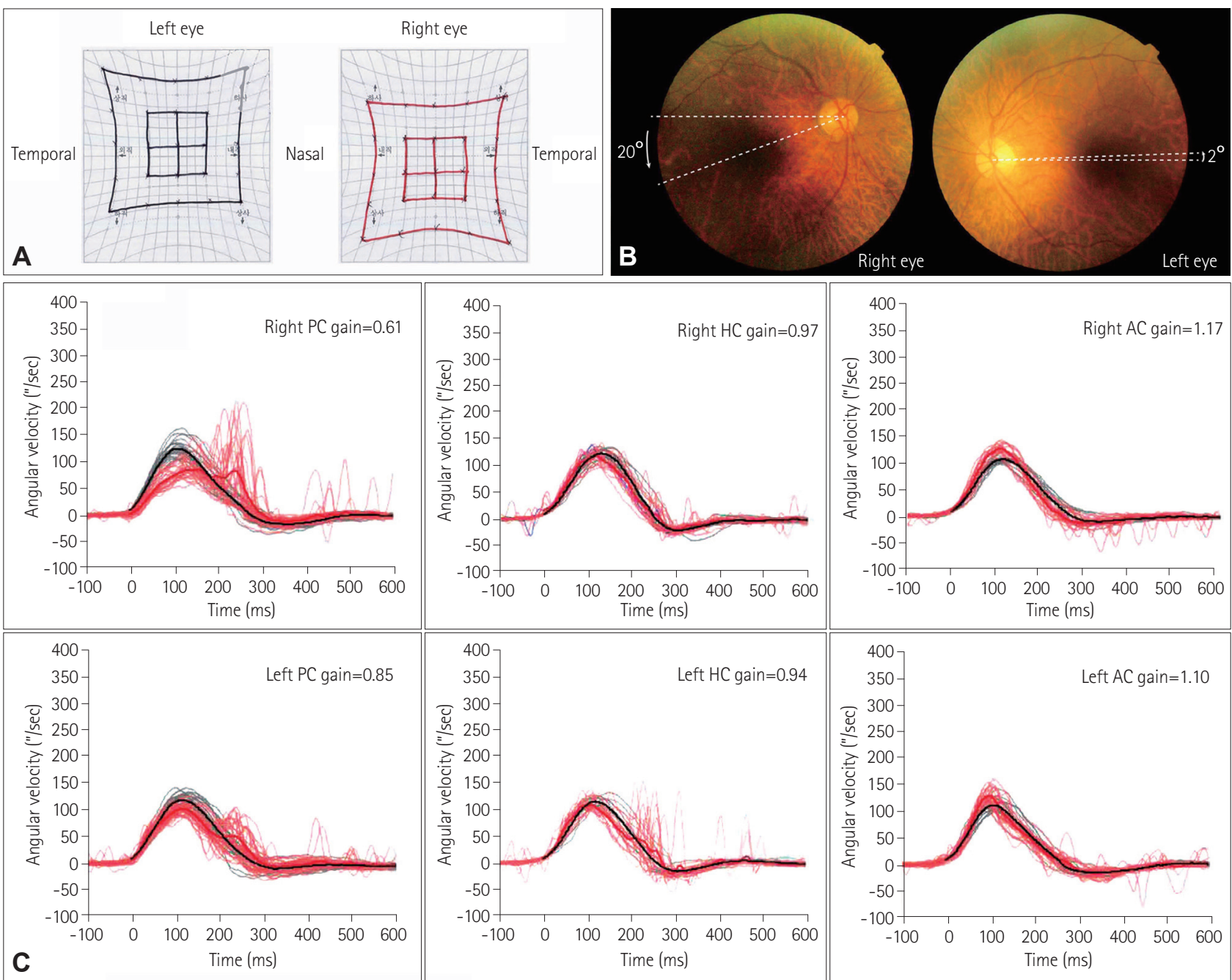

Fig. 1. Evaluation of diplopia, ocular torsion and vestibular function tests. A: An ocular motility test with a Hess-Weiss screen showed hypertropia of the left eye (skew deviation to the right). B: Fundus photographs indicating $20^{\circ}$ extorsion of the right eye and $2^{\circ}$ intorsion of the left eye. C: Video head impulse tests showed a decreased vestibulo-ocular reflex gain with corrective catch-up saccades of the right PC (normal gain=0.881.06), but normal gains for the left posterior and both the $\mathrm{AC}$ (normal gain=0.91-1.07) and the HC (normal gain=0.88-1.03). D: Cervical vestibularevoked myogenic potentials showed symmetrical responses (an interaural difference at 16\%, normal range $<22 \%$ ). AC: anterior semicircular canal, HC: horizontal semicircular canal, LE: left ear stimulation, PC: posterior semicircular canal, RE: right ear stimulation. 
initial and follow-up MRI, and the decreased VOR gain of the unilateral PC in our patient suggest the presence of partial APV selectively affecting the utricle and PC. Cases with partial APV selectively affecting vestibular structures have been recently reported. ${ }^{4-6,11}$

Both skew deviation and trochlear nerve palsy present with hypertropia in the primary position. Incyclotorsion of the hypertropic eye supports skew deviation rather than trochlear nerve palsy, since unilateral trochlear nerve palsy results in hypertropia and the affected eye being unable to incyclotort. ${ }^{12}$ Furthermore, the SVV of the patient was tilted toward the contralateral side of the hypertropia. ${ }^{13}$

\section{Conflicts of Interest}

The authors have no potential conflicts of interest to disclose.

\section{Acknowledgements}

This work was supported by a 2-year Research Grant of Pusan National University.

\section{REFERENCES}

1. Baloh RW. Clinical practice. Vestibular neuritis. N Engl J Med 2003;348: 1027-1032.

2. Halmagyi GM, Weber KP, Curthoys IS. Vestibular function after acute vestibular neuritis. Restor Neurol Neurosci 2010;28:37-46.
3. Kim JS, Kim HJ. Inferior vestibular neuritis. J Neurol 2012;259:15531560.

4. Manzari L, MacDougall HG, Burgess AM, Curthoys IS. Selective otolith dysfunctions objectively verified. J Vestib Res 2014;24:365-373.

5. Park JY, Choi SY, Choi JH, Choi KD. Vestibular neuritis selectively involving posterior canal and utricle. J Neurol 2018;265:1940-1942.

6. Taylor RL, McGarvie LA, Reid N, Young AS, Halmagyi GM, Welgampola MS. Vestibular neuritis affects both superior and inferior vestibular nerves. Neurology 2016;87:1704-1712.

7. Kattah JC, Talkad AV, Wang DZ, Hsieh YH, Newman-Toker DE. HINTS to diagnose stroke in the acute vestibular syndrome: three-step bedside oculomotor examination more sensitive than early MRI diffusion-weighted imaging. Stroke 2009;40:3504-3510.

8. Wolfe GI, Taylor CL, Flamm ES, Gray LG, Raps EC, Galetta SL. Ocular tilt reaction resulting from vestibuloacoustic nerve surgery. Neurosurgery 1993;32:417-420; discussion 420-421.

9. Safran $A B$, Vibert D, Issoua D, Häusler R. Skew deviation after vestibular neuritis. Am J Ophthalmol 1994;118:238-245.

10. Vibert D, Häusler R, Safran AB, Koerner F. Diplopia from skew deviation in unilateral peripheral vestibular lesions. Acta Otolaryngol 1996; 116:170-176.

11. Tarnutzer AA, Bockisch CJ, Buffone E, Weber KP. Association of posterior semicircular canal hypofunction on video-head-impulse testing with other vestibulo-cochlear deficits. Clin Neurophysiol 2017;128: 1532-1541.

12. Wong AM. Understanding skew deviation and a new clinical test to differentiate it from trochlear nerve palsy. J AAPOS 2010;14:61-67.

13. Hernowo A, Eggenberger E. Skew deviation: clinical updates for ophthalmologists. Curr Opin Ophthalmol 2014;25:485-487. 